\title{
Trabajos (in)decentes en la explotación de la caña de azúcar en el área geográfica del río Cauca, Colombia*
}

\author{
(In) decent jobs in the exploitation of sugar cane \\ in the geographical area of the Cauca River, Colombia
}

Cristian Bedoya Dorado*

Rec: $10 / 12 / 2019$ Acep: 15/04/2020

\section{Resumen}

En este artículo se analizan las condiciones de trabajo en la fase agrícola de la caña de azúcar en el área geográfica del río Cauca. La discusión se plantea desde la noción de "Trabajo decente y crecimiento económico" de la Agenda 2030 y los Objetivos de Desarrollo Sostenible (ODS). Desde un enfoque cualitativo, se desarrolló una investigación de diseño etnográfico multilocal, se realizaron entrevistas semiestructuradas y diarios de campo. Las condiciones de trabajo se caracterizan por ser precarias, inestables, informales, intensificadas, inciertas y de explotación, lo que configura trabajos "indecentes" en el contexto de un sector azucarero que genera desarrollo económico y de posicionamiento en el mercado internacional. Pese a que la precarización en el trabajo agrícola de este sector ha sido una realidad históricamente, se intensificó debido al declive de las oportunidades de trabajo, producto de su reemplazo por el uso intensivo de máquinas.

Palabras clave: sector azucarero; trabajo decente; precarización laboral; corteros de caña, agricultura.

\begin{abstract}
This article analyses the working conditions in the agricultural phase of sugar cane in the geographic area of the Cauca River. The discussion arises from the notion of "Decent work and economic growth" of the 2030 Agenda and the Sustainable Development Goals (SDG). From a qualitative approach, multilocal ethnographic design research was developed, semi-structured interviews and field diaries were conducted. The working conditions are characterized by being precarious, unstable, informal, intensified, uncertain and exploitative, which configures "indecent" jobs in the context of a sugar sector that generates economic development and positioning in the international market. Even though precariousness in agricultural work in this sector has historically been a reality, it intensified due to the decline in job opportunities, as a result of their replacement by the intensive use of machines.
\end{abstract}

Keywords: sugar sector; decent job; job insecurity; cane cutters, agriculture.

* Artículo producto de investigación, proyecto "Trabajos 'amargos' en una tierra 'dulce': afectos de Corteros de Caña en el Valle del Cauca”. Unicatólica

** Administrador de Empresas, magíster en Psicología Universidad del Valle. Docente e investigador del Grupo de Investigación (Gilco) Facultad de Ciencias Empresariales de la Fundación Universitaria Católica Lumen Gentium. Correo electrónico: cbedoya@unicatolica.edu.co; Orcid: https://orcid. org/0000-0001-9609-0319 


\section{Introducción}

El departamento del Valle del Cauca ha sido históricamente un epicentro de la agricultura colombiana, caracterizado principalmente por la explotación de la caña de azúcar. En la actualidad, la caña para la producción de azúcar y panela es el segundo generador de empleo después del café en Colombia, según datos de Fedesarrollo del 2019, el sector agroindustrial genera más de 286.000 empleos directos, indirectos e inducidos en los que el $65 \%$ de las familias en los municipios productores de la caña (exceptuando Cali y Pereira) están vinculados a la agroindustria (Asocaña, 2019a, 2019b).

No obstante, en 2008 las condiciones de los corteros de caña y otros trabajadores agrícolas de este producto se pusieron de manifiesto mediante distintos modos de protesta (Montoya, 2011). Entre las necesidades e inconformidades de los trabajadores se encontraba el pago de salarios inferiores al salario mínimo legal vigente, inestabilidad laboral, jornadas de trabajo de más de diez horas, atropellos y castigos por no trabajar debido a enfermedades o lluvias, entre otras más (Montoya, 2011). No obstante, el sector azucarero se encontraba respaldado porque las responsabilidades legales-patronales no recaían sobre ellos sino sobre las Cooperativas de Trabajo Asociado (en adelante CTA). Este modelo para la subcontratación y tercerización de la mano de obra tiene sus antecedentes en la Ley 50 de 1990, que propicia los procesos de flexibilidad laboral y los Decretos 468 de 1990 y 4588 de 2006 que definen las CTA y su objeto social. Las CTA vinculaban y pagaban los salarios y prestaciones sociales a los trabajadores agrícolas de la caña de azúcar (Montoya, 2011; PérezRincón y Álvarez-Roa, 2009).

Este fenómeno ha sido objeto de diversas investigaciones y estudios como los de Giraldo (2017), Jaramillo (2017), Montoya (2011), PérezRincón y Álvarez-Roa (2009), Ronderos y Palacios (2010) y Sánchez (2008), en los que se examinan características tales como: precarización laboral, subcontratación y tercerización, inestabilidad en el trabajo, intensificación del trabajo, violación de derechos humanos, entre otros. Estas características no solo dibujan el contexto del trabajo y de las organizaciones (Bedoya, 2017) sino que además, configura un proceso histórico de los resultados de las políticas del neoliberalismo; la globalización; la decadencia del Estado de bienestar; la expansión de la caña de azúcar y la apuesta por el monocultivo; los ejercicios de poder del sector azucarero y sus estrategias de competitividad basadas en la reducción de costos (nomina directa, las relaciones de trabajo establecidas en el Código Sustantivo de Trabajo se trasladaron al Código de Comercio, en el cual aparecieron las (TA), etc.

Con ello, los trabajadores agrícolas de la caña de azúcar se convirtieron en los más precarios y explotados en toda la cadena productiva de la caña de azúcar. Autores como Pérez-Rincón y ÁlvarezRoa (2009) cuestionan las "condiciones dignas" de estos trabajadores, mientras que Giraldo (2017) encuentra que, para ellos, sus condiciones no solo afectan la vida productiva, sino también la social. Es por ello que para Jaramillo (2017), los trabajadores agrícolas, a través del Sindicato Nacional de Corteros (Sinalcorteros), continúan en la reivindicación de los derechos laborales, buscan una contratación directa, estabilidad laboral y negociaciones colectivas.

Pese a lo anterior, desde el 2007 el sector azucarero se adhirió al Pacto Global (Dinero, 2007), y en los informes de sostenibilidad de algunos ingenios se estipula el modo para orientarse a los objetivos de la Agenda 2030 (Bedoya, 2019). Asimismo, dichos informes evidencian las prácticas de RSE que trabaja este sector durante los últimos años, en materia de desarrollo humano y social (e.g., Ingenio Carmelita, Manuelita, María Luisa, Mayagüez, Pichichí, Sancarlos, Riopaila-Castilla, y Providencia) (Bedoya, 2019).

Uno de los temas más significativos en materia de condiciones de trabajo se encuentra en la Agenda 
de trabajo decente (OIT, 2019a; 2020). La noción de trabajo decente fue propuesta en la Cumbre Mundial para el Desarrollo Social en 1995 como medio para promover la creación de empleos sostenibles. En los últimos años, esta noción se adoptó en la Agenda 2030 para el Desarrollo Sostenible y los nuevos Objetivos de Desarrollo Sostenible (ODS) del Clobal compact o Pacto Global de la ONU (Sustainable Develpment Goals Fund [SDGF], 2019), entre los que se encuentra "Trabajo decente y crecimiento económico" (Objetivo n. 8). Bajo este objetivo se reconoce la inequidad y precarización que existe en trabajos alrededor del mundo, y que en ocasiones, tener trabajo no garantiza salir de la pobreza. Así mismo, se busca crear oportunidades de trabajo decente, crecimiento sólido e inclusivo, y reducir las desigualdades (OIT, 2018; 2019a), al tiempo que se reconocen las particularidades de cada tipo de trabajo como es lo son aquellas del campo y la agricultura (OIT, 2019b; OIT y Monje, 2014)»publisherplace»:»Turín»,»title»:»Trabajo decente, Pobreza y Empleo Agrícola. Experiencias de organización sindical en el sector rural frente a la informalidad en América Latina.»,»type»:»bbook»),»uris»:[«http:// www.mendeley.com/documents/?uvid=e9cf7377422c-4162-9429-f115be7916c0»]\},\{《id»:»ITEM2»,»itemData»:\{《abstract»:»La agricultura es una importante fuente de empleo e ingresos, en particular en los países en desarrollo. Sin embargo, los trabajadores agrícolas suelen enfrentar déficits de trabajo decente, trabajar por un salario y unos ingresos escasos en condiciones a veces peligrosas, y carecer de medios para abordar su situación de forma eficaz. Lograr el trabajo decente y mejorar la productividad en la agricultura son dos requisitos fundamentales para reducir la pobreza y estimular el crecimiento económico en las zonas rurales. A fin de satisfacer esos requisitos, es crucial aumentar el número de empleos y mejorar su calidad, promover los derechos en el trabajo, ampliar la protección social y fortalecer las organizaciones de trabajadores rurales. Para alcanzar estos objetivos, el enfoque de la Organización Internacional del Trabajo (OIT.
El presente artículo tiene como finalidad analizar el trabajo agrícola de la caña de azúcar en el área geográfica del río Cauca a la luz de la categoría "trabajo decente", se estructura en tres apartados. Primero se explica la propuesta metodológica para el análisis de las condiciones de trabajo en la fase agrícola de la caña de azúcar. En segunda instancia se discuten los resultados a la luz de la categoría trabajo decente. Finalmente, se presentan conclusiones y recomendaciones para futuras líneas de investigación.

\section{Diseño metodológico}

El ejercicio empírico se circunscribe en un enfoque cualitativo (Guba y Lincoln, 1994; Willig, 2001). La necesidad de conocer las experiencias de trabajos agrícolas de distintos sujetos asociados requirió realizar trabajo de campo en múltiples localidades y aplicar diversas estrategias de recolección de la información. Es por ello que la investigación tuvo un diseño etnográfico (Flick, 2007) en el que se utilizó la etnografía multilocal, la cual se desarrolla alrededor de cadenas, sendas, tramas, conjunciones o yuxtaposiciones de locaciones. En ella el investigador define el modo de su presencia bajo una lógica que explica la asociación y conexión entre los escenarios que definen el argumento de la etnografía. La etnografía multilocal permite además definir los objetos de investigación a partir de diversas modalidades o técnicas para el rastreo de personas, objetos, signos, símbolos y metáforas (Falzon, 2009; Marcus, 2001).

Las estrategias de recolección de la información empleadas fueron: la observación participante de tipo "observador como participante", con la cual se registraron notas de campo; la entrevista en la modalidad semiestructurada; y el grupo focal orientado a tres temas de discusión: 1) trabajos agrícolas de la caña de azúcar y condiciones de trabajo; 2) transformaciones geográficas y de relaciones laborales; y 3) trabajo agrícola desde una perspectiva de género y de la edad (Falzon, 2009; 
Flick, 2007; Mayan, 2009; Willig, 2001). La recolección de la información se realizó en distintas localizaciones del área geográfica del río Cauca en el Valle del Cauca y norte del Cauca, se agrupa en tres tipos de escenarios: (1) la jornada de trabajo en los cultivos de caña; (2) el final de la jornada de trabajo en los cultivos de caña y en las empresas dedicadas a la explotación de la caña de azúcar; y (3) en los lugares de residencia de los trabajadores.

Los participantes se seleccionaron por conveniencia y según su voluntad para participar en el estudio (Mayan, 2009). Como criterios de inclusión se tuvieron en cuenta: ser mayores de edad y trabajar o haber trabajado en la fase agrícola de la explotación de la caña de azúcar en el área geográfica del río Cauca. Algunas de las localidades se definieron durante los primeros trabajos de campo, con el objetivo de rastrear a los trabajadores agrícolas de la caña de azúcar.

La cantidad de participantes estuvo determinada por la estrategia de muestra tiempo-locación, en la que las entrevistas se realizan en una localización específica durante un tiempo en particular (Patton, 2015), en este caso: las fechas definidas de trabajo de campo, los horarios de jornada de trabajo, la finalización de trabajo de campo y los días de descanso en los hogares de los participantes. Este mismo criterio se empleó para la organización y cantidad de grupos focales. Con relación a los aspectos éticos de la investigación, a los participantes se les entregó un consentimiento informado y se les explicó el objetivo de la investigación, así como la protección de la información suministrada para uso exclusivamente académico.

Las notas de campo, las trascripciones de las entrevistas y de los grupos focales se analizaron bajo la noción de trabajo decente para el trabajo rural y agrícola, la cual se basa en cuatro pilares: empleabilidad, protección social, estándares y derechos en el trabajo, y gobernanza y diálogo social (FAO, 2013; Oya, 2015). Mediante una categorización abierta y axial (Strauss y Corbin, 2002) se utilizaron como categorías de análisis los indicadores empleados por la OIT y la FAO para analizar el trabajo decente (Anker et al., 2002; FAO, 2013; OIT, 2013, 2018; Oya, 2015), y se abordaron atributos como la informalidad y flexibilización del trabajo en tanto fundamentos de la precarización laboral (Antunes, 2000; Soto, 2008; Soto et al., 2008). A manera de apoyo a la validez metodológica se utilizó la propuesta de Saukko (2012) centrada en la realidad social (dimensión contextual), realidades locales (dimensión dialógica) y modelación social de la realidad (dimensión autorreflexiva). En la siguiente tabla se resume el diseño metodológico de la investigación: 
Cristian Bedoya Dorado

Tabla 1. Diseño metodológico

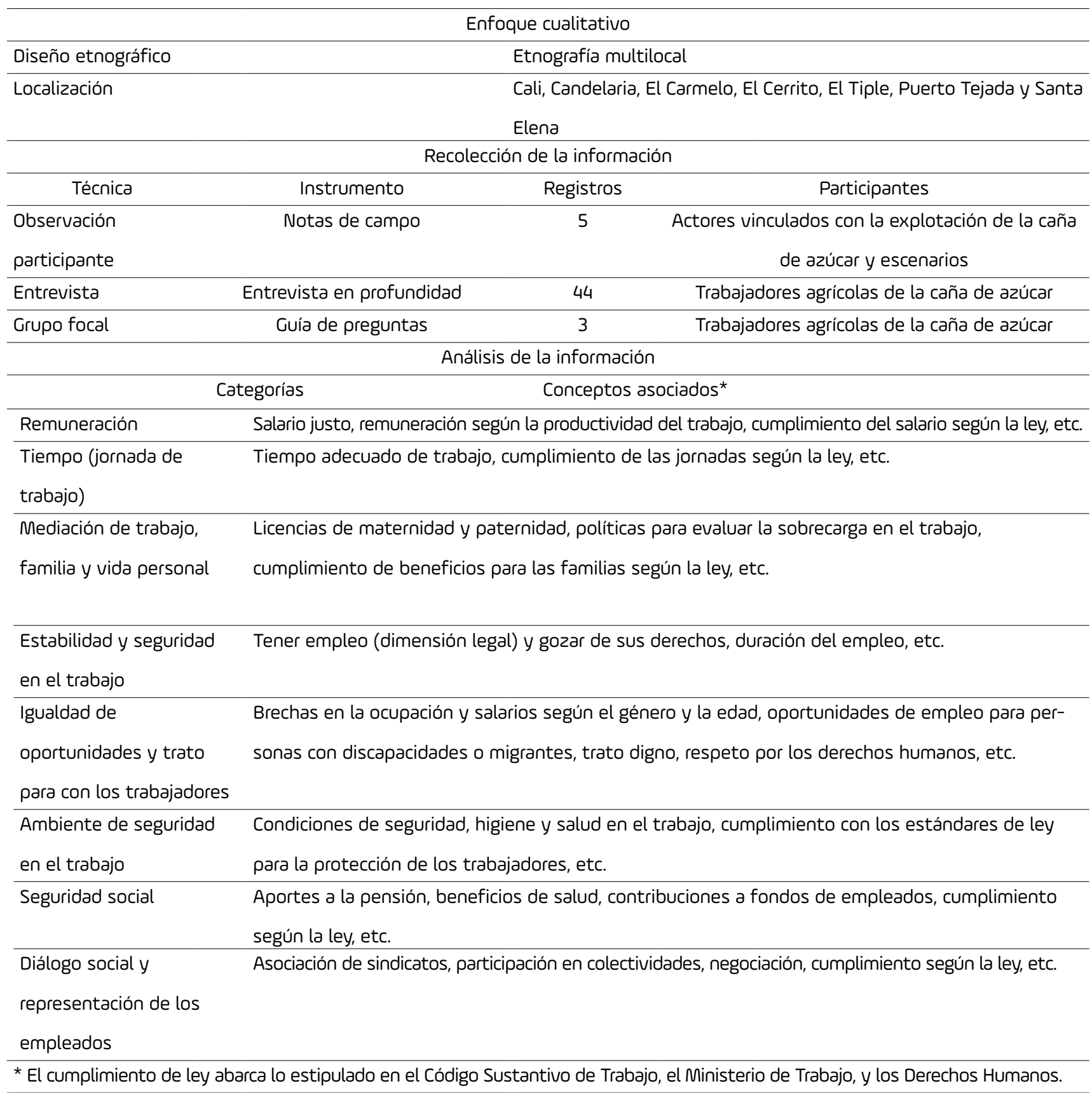

Fuente: elaboración propia 


\section{Trabajos agrícolas de la caña de azúcar}

\section{Características sociodemográficas de los participantes}

Del total de participantes de las entrevistas, 10 eran mujeres y 34 hombres. La edad promedio fue de 57 años, en un rango que va desde los 30 hasta los 84 años. Las trayectorias de trabajo con la caña de azúcar en su fase agrícola son en promedio de 26 años, van desde los 4 hasta los 60 años. Los participantes viven, en su mayoría, en el departamento del Cauca ( $n=26)$ en las ciudades de Guachené, Puerto Tejada, Santander de Quilichao, Timbiquí, y Toribío, el resto habitan en ciudades del Valle del Cauca $(n=18)$ como Candelaria, El Carmelo, Palmira, Pradera y Villagorgona. en los diarios de campo se registró un predominio de trabajadores afrocolombianos $(n=42)$ originarios del Pacífico colombiano (Cauca, Chocó y Valle del Cauca) y norte del Cauca.

Los participantes desempeñan actividades agrícolas alrededor de la caña de azúcar como alzadores, quienes ponen la caña de azúcar cortada en los vagones ( $n=2)$; cabos organizan el trabajo de la fase agrícola de la caña de azúcar, principalmente el corte y el alce de la caña, lo que involucra las suertes distribución de los cultivos- que cada trabajador debe cortar, y luego subir ( $n=2)$; caseros y oficios varios, encargados de cuidar una finca y los cultivos de caña de azúcar de los dueños de las tierras -sean estos colonos o los ingenios- y realizar labores agrícolas en los cultivos ( $n=1)$; contratistas, encargados de vincular a los trabajadores agrícolas de la caña de azúcar. Tanto este trabajo como el del cabo, se asocian a funciones administrativas y organizativas dentro de la fase agrícola de la caña de azúcar ( $\mathrm{n=}$ 1); cortadores de semilla quienes cortan nuevamente la caña de azúcar -ya cortada- en pequeños trozos, en promedio de 10 a $30 \mathrm{~cm}$, y que se utilizan para la siembra ( $n=6)$; corteros de caña $(n=27)$; y oficios varios, incluye actividades como el corte y alce de la caña, macollar (retirar la maleza de los cultivos), palar para organizar la siembra, abonar y fumigar, ayudar en los procesos de riego (según el método utilizado por los dueños de las tierras), recortar luego del corte con máquina, resembrar, etc. $(n=5)$.

Las mujeres realizan principalmente actividades como el corte de semilla ( $n=6)$, mientras que los hombres laboran principalmente como corteros de caña ( $n=27)$. La mayoría de los participantes trabajan de manera informal y están vinculados por medio de un "contratista" ( $n=39$ ), algunos de estos son pensionados ( $n=7)$. Los demás participantes trabajan bajo condiciones de formalidad, en las modalidades de subcontratación ( $n=2)$ y contrato directo con empresas del sector azucarero $(n=3)$.

\section{Categorías de análisis}

Las características del trabajo de los participantes dependen y difieren principalmente debido al tipo de contrato y las modalidades de trabajo. Es por ello que la mayoría de las características de trabajo decente, como se presentan a continuación, se ven determinadas por la formalidad del trabajo (ser empleado directo o estar subcontrato), mientras que en la informalidad se evidencia su ausencia.

\section{Remuneración}

Los relatos de los participantes que tienen una vinculación directa con las empresas del sector azucarero o que son subcontratados por estas evidencian, no solo un cumplimiento del marco normativo con relación al salario mínimo legal vigente (SMLV) (para el 2019 el SMLV se fijó en \$ 828.116, con auxilio de transporte en \$ 925.148), sino que además, algunos tienen remuneraciones superiores a este, debido a que hay temporadas en las que las empresas demandan jornadas de trabajo extra (principalmente los domingos), que permiten obtener pagos adicionales (diario de campo, 2019, septiembre 28). No obstante, algunos de estos trabajadores formales manifiestan que la remuneración está muy cercana al SMLV y que no es suficiente para solventar los gastos de sus familias, las cuales son numerosas (en promedio cuatro integrantes) y dependen de un solo ingreso económico. Lo anterior se refleja también 
en algunos casos como los de los trabajadores jubilados de empresas del sector azucarero, que deben continuar trabajando de manera informal para aumentar los ingresos económicos de sus familias (diario de campo, 2019, septiembre 13). Finalmente, debido al esfuerzo físico que exige el trabajo agrícola de la caña de azúcar, principalmente el corte y alce de la caña, algunos participantes consideran que la remuneración es muy baja (diario de campo, 2019, septiembre 27).

En el caso de los trabajadores informales, o "piratas", como ellos mismos denominan (comunicación personal, participante 40, mujer cortera de caña y semilla, 49 años, octubre 13), la remuneración es un asunto mucho más complejo por diversos asuntos. Por una parte, esta depende estrictamente del nivel de productividad de los trabajadores y se fija de acuerdo con las tareas agrícolas que realizan.

En estas tierras de caña de azúcar, [a] un cortero le pagan $\$ 11.600$ pesos por tonelada de caña cortada. Según ellos, el que más rinde puede llegar a cortar hasta 4 toneladas por día, lo que significa que en la semana podría ganarse hasta $\$ 232.000$. Sin embargo, esto es algo que sucede de manera esporádica, pues los corteros más productivos ganan alrededor de $\$ 200.000$ por semana. (diario de campo, 2019, septiembre 27)

Al ser la remuneración en el trabajo informal un asunto de la productividad, aparecen en juego variables como las de género. Para la mayoría de los participantes existe poca presencia de mujeres en estos trabajos por la fuerza y resistencia física que requiere, por lo que se considera que es un trabajo para "machos". Esto se evidencia en los ejercicios de observación, en los que las mujeres realizan actividades como abono, siembra, despajo, macollar, entre otras actividades agrícolas (diarios de campo, 2019, septiembre, 13 y 27, octubre 13). A pesar de ello, el rastreo de los trabajadores agrícolas de la caña de azúcar permitió develar que existen mujeres corteras de caña que se enfocan principalmente en el corte de semilla, muchas de ellas habitan en el norte del Cauca, en especial en Puerto Tejada:

A míme pagan lo que yo haga con un contratista. ¿Cómo es la forma de pago con el contratista? La cantidad de paquetes. El paquete vale \$ 220 pesos, en un promedio se hace 200 al día. Nos pagan cada 15 días. (comunicación personal, participante 28, mujer cortadora de semilla, 50 años, octubre 20)

Por otra parte, la dependencia de la remuneración según la productividad de los trabajadores hace que los ingresos puedan estar por encima del SMLV, sin embargo, se evidencian condiciones físicas que hacen que, en la mayoría de los participantes, esta esté por debajo de dicho parámetro legal. Por ejemplo, de acuerdo con las observaciones la mayoría de los corteros de caña son adultos mayores (diarios de campo, septiembre 13, 27 y 29, octubre 13), eso se refleja en las características sociodemográficas, en las que las edades promedio son 57 años, factor que hace más compleja la productividad y por ende la remuneración. Así mismo, se observan personas en situación de discapacidad física y visual que obtienen remuneraciones aproximadamente entre $\$ 80.000$ a $\$$ 120.000 semanales (diarios de campo, septiembre 13, 27 y 29 , octubre 13).

Al depender la remuneración de la productividad en el trabajo informal, si los trabajadores no se presentan a los cultivos por motivos de enfermedades, condiciones climáticas $u$ otras, no existe ningún tipo de ingreso (diario de campo, 2019, septiembre 27). Algunos participantes (tanto formales como informales) consideran que no existe justicia en la remuneración de los trabajos agrícolas de la caña de azúcar, esto se ve reflejado en las condiciones de pobreza y vulnerabilidad en las que viven, y en la reproducción de ciclos de pobreza en sus familias, quienes con dificultad tienen acceso a educación, 
salud y condiciones de vida dignas (diarios de campo, 2019, septiembre 29, octubre 13 y 20).

\section{Tiempo (jornada de trabajo)}

En las labores formales las jornadas de trabajo giran alrededor de las 8 horas, los horarios son entre las 6:00 a.m., y las 3:00 p.m., de lunes a viernes. Sin embargo, según las demandas de caña de azúcar de las empresas, citan a los trabajadores a que se presenten a realizar actividades agrícolas en otros horarios: "Si en este momentico me llaman y me dicen que debo estar a las diez de la noche, debo hacerlo" (comunicación personal, participante 27, hombre cortero de caña, 56 años, septiembre 27).

En los trabajos informales se presenta una similitud con los formales, puesto que las jornadas de trabajo se realizan principalmente entre las 6:00 a.m., y las 3:00 p.m. Esto se debe a que las condiciones climáticas en el trabajo agrícola de la zona hacen que, además de la fuerza y resistencia física, los trabajadores deban soportar el sol y las altas temperaturas que oscilan entre los $19^{\circ}$ y $30^{\circ}$, aunque pueden llegar a estar por encima de los $32^{\circ}$. Así mismo, las temporadas de lluvias constituyen un factor decisorio sobre si se trabaja o no, o el tiempo de trabajo en el caso de los trabajadores informales (diarios de campo, 2019, septiembre 13 y 27).

Si bien en los trabajos formales las empresas del sector azucarero pueden planificar y organizar trabajos agrícolas en jornadas diferentes a las normales, en los trabajos informales esta condición está determinada por la disponibilidad del transporte, lo que caracteriza la flexibilidad del trabajo agrícola bajo este tipo de vinculación. El contratista que se encarga de suplir la mano de obra para los trabajos agrícolas contrata un transporte que con unas rutas específicas recoge a los trabajadores y los regresa a sus hogares. La organización de estas rutas determina las jornadas de trabajo, sin embargo, si los trabajadores disponen de algún medio de transporte, tienen la posibilidad de ampliar sus jornadas de trabajo que podrían ser de hasta diez horas diarias (diarios de campo, 2019, septiembre13 y 27; grupo focal, 2019, septiembre 29):

Yo vivo en Gorgona. A media, hora y media. [... Yo ando en cicla [...] Pues aquí entra uno a las 6:00 a.m., trabaja lo que uno pueda, trabaja hasta la 1:00, 1:30, 2:00 de la tarde. Esa es la jornada de todos nosotros, de todos los corteros (comunicación personal, participante 2, hombre cortero de caña, 60 años, septiembre 13).

\section{Mediación trabajo, familia y vida personal}

Las jornadas de trabajo, tanto para los trabajadores formales como informales, si bien se encuentran en el margen del promedio de horas que se trabajan legalmente en Colombia (ocho horas), no son determinantes en la mediación del tiempo de trabajo y la vida familiar y personal, aunque si lo es el tiempo que se requiere para llegar a los cultivos de la caña de azúcar y de regresar a los hogares. Lo anterior se suma al tiempo que se requiere para prepararse para ir al trabajo (e.g. preparación de alimentos, baño, vestuario, elementos de trabajo, etc.). Se encuentra que algunos participantes requieren en promedio entre una y dos horas para llegar a sus sitios de trabajo y regresar de él, ello depende de las rutas del transporte del contratista o de las empresas, o de sus propios medios de transporte (diario de campo, 2019, septiembre 13 y 27, octubre 13).

En este contexto el soporte que dan las mujeres a los hombres trabajadores con las labores domésticas es de gran importancia, hacen que los tiempos de preparación para irse a sus trabajos sean inferiores a los requeridos cuando el hombre no tiene cónyugel y así disponen de más tiempo para dormir, la familia y actividades de la vida personal: "La mujer mía se levanta a las tres de la mañana. - ¿Y usted?- A las cuatro de la mañana [...] Porque ellas ayudan con lo

1 Se utiliza esta palabra para referenciar a la mujer pareja del trabajador que vive con él, independientemente de su estado civil. En los discursos y retórica de los participantes, a ellas se les denomina "la mujer" o "mi mujer". 
de la alimentación. [...] Si, ellas cocinan" (comunicación personal, participante 13; hombre cortero y alzador de caña, 43 años, septiembre 29).

No obstante, en el caso de las mujeres esta situación es más compleja, ellas mismas realizan las actividades domésticas previo al trabajo, lo que implica doble jornada de trabajo:

[...] a las cuatro yo madrugo. -¿Cómo se desplaza al lugar?- En bus, del municipio. -¿Cuánto se demora?- Más o menos una hora. - ¿Usted le toca levantarse a preparar la alimentación?- Yo dejo las ollas hirviendo, me voy a bañar y salgo y ya está. -¿Cuántas comidas lleva?- Solo el almuerzo, y el desayuno compro un pan [...] Llevo agua, aguapanela y café. - ¿A qué horas empieza a trabajar?- A las 8 a.m., uno trabaja hasta las 4 p.m., de lunes a sábado. (comunicación personal, participante 28; mujer cortadora de semilla; 50 años, octubre 13)

\section{Estabilidad y seguridad en el trabajo}

Pese a que a diversas condiciones para tener un "trabajo decente" están mediadas por el tipo de contrato y la modalidad de empleo de los participantes, el fenómeno de la estabilidad y seguridad en el trabajo se asocia a factores subjetivos. Comentaron algunos participantes que por responsabilidad social de las empresas, no se pueden despedir a todos los trabajadores agrícolas de la caña azúcar, en el marco de una amenaza de reemplazo de sus trabajos por máquinas. Esto conlleva que algunas empresas capaciten y reubiquen a los trabajadores contratados en otro tipo de labores, de modo que no se vean afectados (diario de campo, 2019, septiembre 13).

Otros participantes consideran que los pequeños productores de los derivados de la caña de azúcar -como la panela- no cuentan con los recursos financieros suficientes para incorporar maquinaria en la fase agrícola de los cultivos, lo que hace necesario que continúen vinculando trabajadores a los cultivos de caña, independiente de la modalidad de empleo. Así mismo, el monocultivo de la caña de azúcar y la consolidación del sector azucarero lleva a que se experimente estabilidad y seguridad: "[...] este trabajo es estable. Llueva, truene o relampaguee hay trabajo" (comunicación personal, participante 13; hombre cortero y alzador de caña, 43 años, septiembre 27).

Los participantes también reconocen que en el trabajo informal es posible tener estabilidad debido a que el pago es muy bajo y no existen muchas personas que estén dispuestas a trabajar bajo esta condición (diario de campo, 2019, septiembre 27). Así mismo, algunos participantes consideran que es posible tener mayor estabilidad y seguridad bajo la modalidad informal que la formal, pues muchos de ellos fueron despedidos por empresas en las que trabajaban con vínculo directo (diario de campo, 2019, septiembre 29).

En el caso de los trabajadores vinculados de manera directa, adicional a la evidencia de despidos también se presentan casos en los que las empresas negocian las indemnizaciones para terminar contratos a término indefinido:

En los ingenios compran las fichas, por ejemplo, a los que llevan tiempo lo llaman a arreglo, le dan veinte o treinta millones de pesos [...] la ficha es que le compran su cupo de trabajo. Por ejemplo, usted trabaja aquí, le dicen le damos tantos millones, usted los recibe y se va. Eso hacen en los ingenios [...] eso no lo reemplazan más [...] ellos no reemplazan a nadie más" (comunicación personal, participante 13; hombre cortero y alzador de caña, 43 años, octubre 13).

\section{Igualdad de oportunidades y trato para con los trabajadores}

Los trabajos agrícolas de la caña de azúcar presentan una característica organizativa con relación a los tipos de trabajo según el género. En el trabajo de campo se pudo evidenciar baja participación de 
las mujeres (diarios de campos, 2019, septiembre13, 27 y 29). Lo anterior, adicional al asunto de los roles sociales que desempeñan, como el cuidado doméstico del hogar para que sus esposos puedan trabajar y las características de una cultura machista insertas en los sistemas sociales en los que viven, las limitan para trabajar, en algunas empresas no se permite que ellas trabajen (diarios de campo, 2019, septiembre 13 y 27).

Las mujeres que trabajan en este contexto logran acceder por la ausencia de la figura masculina en los hogares o por la necesidad de aumentar los ingresos de las familias (grupo focal, 2019, octubre 13). Las mujeres entrevistadas relatan que son pocas y en algunos casos, son las únicas mujeres entre el grupo de trabajadores, se les ubica principalmente en actividades del corte de semilla: "[...] la mujer no la pone a trabajar, son amas de casa. Y habemos mujeres que trabajan más en la casa que los propios maridos. Como me pasa a mí. Donde yo trabajo hay más mujeres cortando semillas" (comunicación personal, participante 40; mujer cortera de caña y semilla, 49 años, octubre 13).

El contexto informal de los trabajos agrícolas de la caña de azúcar ofrece un panorama de oportunidades de trabajo, principalmente para hombres pensionados y adultos mayores. Esto se explica por la consideración de que los hombres cumplen con el criterio de fuerza y resistencia física para este tipo de trabajos y porque a los pensionados y adultos mayores no se cotiza al sistema de pensiones, lo que hace que su vinculación disminuya los costos de los contratistas (diarios de campo, 2019, septiembre 13, 27, 28 y 29, octubre 13).

Finalmente, algunos participantes relatan experiencias de maltrato psicológico y de discursos racistas hacia ellos, así como la violación de derechos humanos y laborales que incluye: despidos sin justa causa, pago de indemnizaciones inferiores a lo que legalmente tendrían derecho, engaño de cotización al sistema de pensiones, amenazas de despido por no querer trabajar durante temporadas de lluvias o por no querer cortar la caña recién quemada, entre otros ejemplos más (grupos focales, 2019, septiembre 29, octubre 13).

\section{Ambiente de seguridad en el trabajo}

Los participantes del estudio consideran que los trabajos agrícolas de la caña de azúcar son riesgosos y peligrosos debido a varias razones: se requieren machetes, pacoras, palas, catana, curvo, entre otras herramientas con las cuales en muchas ocasiones, los trabajadores se cortan o se accidentan. Las posiciones del cuerpo para el corte, el alce, palar, sembrar, etc., afectan algunas partes del cuerpo tales como hombros, cadera, espalda, rodillas, muñecas, etc. En algunos cultivos y pese a estar prohibido, todavía se utiliza el sistema de quema de caña previo a su corte, lo que ocasiona problemas respiratorios. Las jornadas de trabajo, las condiciones climáticas de los cultivos de caña y el esfuerzo físico originan deshidratación y enfermedades de la piel (principalmente para trabajadores que no son afrodescendientes) (diarios de campo, 2019, septiembre 13 y 27).

Para el caso de los trabajadores vinculados directamente a las empresas, las condiciones de seguridad son mejores, algunos reciben capacitaciones sobre manipulación de herramientas y modo de cortar la caña. Así mismo, este tipo de trabajadores reciben dotaciones que incluyen zapatos especiales (botas o guayos), camisas manga larga, gafas protectoras, guantes, pantalón grueso, dulce abrigos, sombreros, machetes y sus variedades, etc. Lo anterior se desdibuja por completo en los trabajadores informales, quienes carecen de todo este tipo de elementos (diarios de campo, 2019, septiembre 27, 28 y 29, octubre 13).

\section{Seguridad social}

El cumplimiento de la seguridad social se evidencia en los participantes vinculados directamente con las empresas del sector azucarero, en el caso de los trabajadores informales se da de manera 
parcial. Como se mencionó anteriormente, a los trabajadores se les cotiza e ingresa al sistema de salud y aseguradoras de riesgos laborales (ARL), pero no al sistema de pensiones, lo que se asocia con los factores sociodemográficos de los participantes y debido a estrategias de disminución de costos de los contratistas o encargados de suplir la mano de obra para los trabajos agrícolas (diarios de campo, 2019, septiembre13 y 27).

Adicional a lo anterior, en sus relatos los participantes contrastan este tipo de beneficios que se reciben en las empresas azucareras bajo vínculos directos o cuando los trabajos son formales (legales) y destacan además, los beneficios de las liquidaciones, primas, vacaciones, cesantías, etc. (grupos focales, 2019, septiembre 29, octubre 13).

\section{Diálogo socialy representación de los empleados}

Ninguno de los participantes con vinculación formal hace parte de sindicatos, sin embargo, algunos de ellos reconocen que las negociaciones y pactos colectivos permitieron que algunos de ellos que trabajaban subcontratados o de manera informal se vincularan directamente en las empresas y obtuvieran otros beneficios como educación, vivienda, préstamos, etc. (grupo focal, 2019, septiembre 13). Así mismo, algunos de los participantes reconocen la importancia que tuvo la huelga del 2008 en Colombia, en la cual participaron y lograron ser escuchados, tanto por las empresas como por el gobierno (grupos focales, 2019, septiembre 29, octubre 13).

Algunas de las razones del paro fueron el atropello de sus derechos:

[...] estuvimos en un paro bastante bravo en el "nombre de un ingenio" porque en ese entonces trabajábamos con contratista y ellos no nos pagaban lo que la empresa mandaba, - sea que la empresa sí mandaba a pagar lo justo, pero entonces qué pasa, que ellos recortaban y se quedaban con lo demás, entonces nosotros debido a eso nos fuimos a paro dos meses. (comunicación personal, participante 14, hombre cortero de caña, 61 años, septiembre 29)

En otros casos esta actividad tuvo consecuencias negativas como el despido:

Pues como en el 2007 o 2008 hubo unos paros de los corteros, entonces yo participé en ellos. De igualmente, los paros se acabaron y seguimos trabajando, pero como allá usan muchas estrategias, con el tiempo a los que éramos líderes buscaron la forma de sacarnos [...] de ahí a casi todos nos sacaron, como en el 2010 nos sacaron. Sacaron como a diez. (comunicación personal, participante 15; hombre cortero de caña, 61 años, septiembre 29)

\section{¿Trabajos decentes o "indecentes"?}

Como se presentó anteriormente, los trabajos agrícolas de la caña de azúcar se caracterizan por coexistir con condiciones decentes e indecentes. En este contexto, la palabra indecente hace alusión a las condiciones de trabajo en las que se presenta la ausencia de las características de trabajo decente impulsadas por la OIT y la FAO, y que, por el contrario, se caracterizan por ser precarias. En la siguiente tabla se resume la presencia de las características de trabajo decente de acuerdo con el vínculo formal e informal de los trabajadores en el sector azucarero: 
Tabla 2. Condiciones de trabajo decente según la vinculación formal e informal

\section{Categoría}

\begin{tabular}{|c|c|}
\hline $\begin{array}{c}\text { Caracteristicas en e } \\
\text { trabajo formal }\end{array}$ & $\begin{array}{c}\text { Caracteristicas en e } \\
\text { trabajo informal }\end{array}$ \\
\hline$\checkmark$ & $x$ \\
\hline$\checkmark$ & $x$ \\
\hline$x$ & $x$ \\
\hline$x$ & $\boldsymbol{x}$ \\
\hline$\frac{v}{x}$ & $\begin{array}{l}x \\
x \\
x\end{array}$ \\
\hline$x$ & $\begin{array}{l}x \\
x\end{array}$ \\
\hline $\begin{array}{l}\boldsymbol{X} \\
\boldsymbol{X} \\
\boldsymbol{X} \\
\boldsymbol{X}\end{array}$ & $\begin{array}{l}x \\
x \\
x \\
x\end{array}$ \\
\hline$\checkmark$ & $x$ \\
\hline$\checkmark$ & $x$ \\
\hline
\end{tabular}

(1) Remuneración

(1) Cumplimiento del SMMLV

(2) Remuneración financiera legal (liquidaciones; primas, vacaciones, cesantías, intereses sobre cesantías, etc.)

(3) Salario justo

(II) Tiempo (jornada de trabajo)

(4) Tiempo adecuado de trabajo

(5) Cumplimiento de la jornada según la ley

(III) Mediación trabajo, familia y vida personal

(6) Licencias de maternidad y paternidad

(7) Evaluación de cargas de trabajo

(8) Beneficios para la familia según la ley

(IV) Estabilidad y seguridad en el trabajo

(9) Estabilidad según el contrato laboral

(10) Derechos laborales

(V) Igualdad de oportunidades y trato para con los trabajadores

(11) Oportunidades en la ocupación según el género

(12) Oportunidades en la ocupación según la edad

(13) Oportunidades de empleo

(14) Trato digno y respeto de los derechos humanos

(VI) Ambiente de seguridad en el trabajo

(15) Condiciones de seguridad, higiene y salud en el trabajo

(16) Cumplimiento con los estándares de la ley para la protección de los trabajadores, etc.

\section{(VII) Seguridad social}

(17) Aportes a la pensión

(18) Beneficios de salud

(19) Fondos para empleados

(20) Administradora de Riesgos Laborales (ARL)

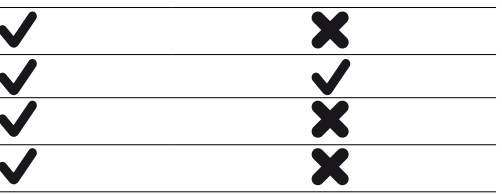

(VIII) Diálogo social y representación de los empleados

(21) Asociación de sindicatos

(22) Participación en colectividades, negociación, cumplimiento según la ley

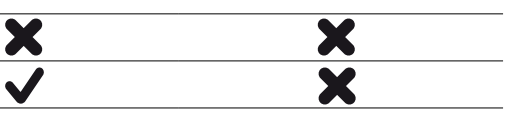

Fuente: elaboración propia 
De acuerdo con lo anterior, el vínculo laboral directo y formal de los trabajadores hace que este tipo de trabajos tengan mayores condiciones de decencia, aunque en algunos casos - por su complejidad y otros factores de gestión-no se presentan. Este es el caso en el que los trabajadores devengan salarios injustos pese al esfuerzo físico que requieren sus tareas; el tiempo inadecuado para desarrollarlas, sumado al tiempo que requieren para la preparación y desplazamiento desde sus casas hasta el lugar de trabajo; la no evaluación de cargas de trabajo para compensar con las jornadas de trabajo, pese a que tienen contratos de trabajo formales, esto no genera una percepción de estabilidad; la experiencia de despidos y compra de puestos de trabajo por parte de las empresas (las fichas) hace que los participantes consideren que estos trabajos son inciertos (inestables). Adicional a lo anterior, para los participantes de este tipo de trabajos existen brechas con relación a las mujeres (baja participación de ellas en los trabajos agrícolas); los participantes de mayor edad tienen mayor probabilidad de despido por la relación fuerza-productividad que a mayor edad disminuye; el remplazo del trabajo manual por el de la máquina hace que la fase agrícola presente pocas oportunidades de empleo; y los relatos de algunos evidencian experiencias de violación de derechos humanos y trato no digno.

En el caso de los trabajadores informales las condiciones de trabajo indecente se maximizan debido a que muchas de estas se impulsan debido a elementos de derechos laborales con los cuales no gozan este tipo de trabajadores. En el caso de características de trabajo decente los participantes consideran que sus jornadas de trabajo son acordes a lo estipulado por la ley, en promedio ocho horas diarias, sin embargo, por depender de la productividad para sus ingresos, muchos deben trabajar jornadas más largas. Por otra parte, a los trabajadores se les vincula al sistema de salud como una de las únicas garantías al sistema de seguridad social.

En la siguiente figura se ilustra la manera en la que las condiciones de trabajo decente e indecente se pueden evidenciar, tanto en los trabajos con vínculo formal (cuadrante 1 y 3) como informal (cuadrante 2 y 4). Los números romanos representan las categorías de la tabla 2.

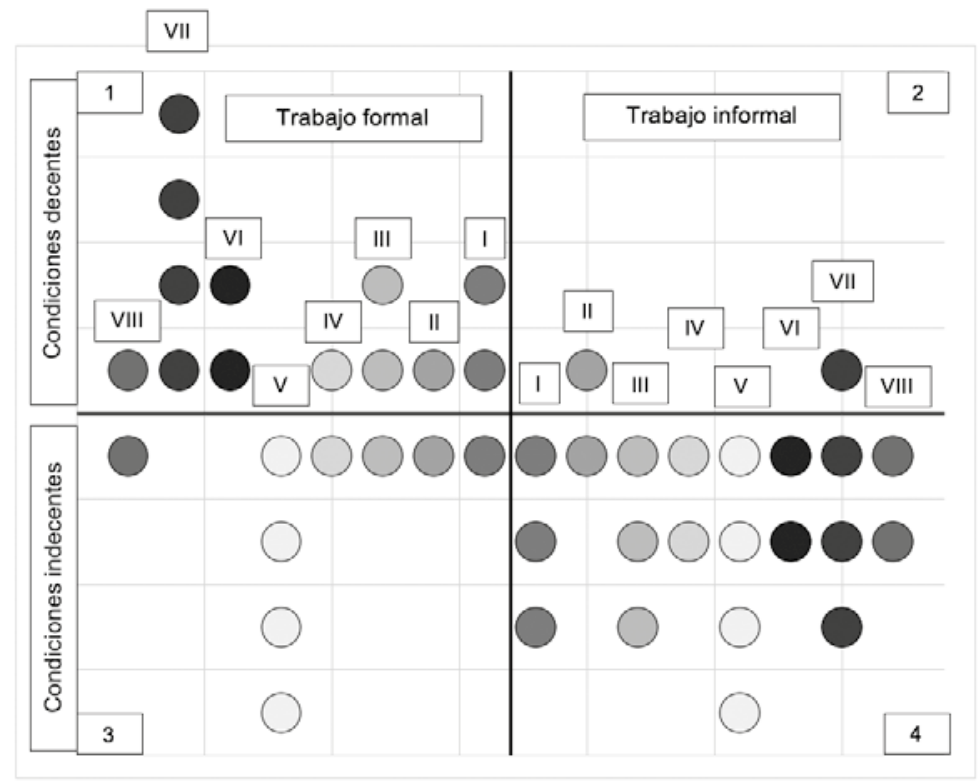

Figura 1. Condiciones decentes e indecentes en los trabajos formales e informales

Fuente: elaboración propia 
Como se mencionó anteriormente, los trabajadores con vínculo formal presentan más características de trabajo decente, como en el caso de la categoría (VII) de Seguridad social, todas se encuentran en el cuadrante 1 de condiciones de trabajo decente, el panorama contrasta con la categoría ( $V$ ) de lgualdad de oportunidades y trato para con los trabajadores, todas son indecentes (cuadrante 3). En el caso de los trabajos informales la mayoría de sus características se ubican en el cuadrante 4.

\section{Conclusiones}

El objetivo del presente artículo fue analizar los trabajos agrícolas de la caña de azúcar que se desarrollan en el área geográfica del río Cauca a la luz de la categoría "trabajo decente". Mediante las estrategias metodológicas para la recolección de información se pudo inferir que este tipo de trabajos son complejos por las condiciones físicas y de resistencia que se requieren en jornadas de trabajo en altas temperaturas y constante exposición al sol, lo que genera desgaste físico. La noción de trabajo decente se reduce en este contexto al cumplimiento de los marcos normativos de la actividad laboral, en este caso el Código Sustantivo de Trabajo. Es por ello por lo que las condiciones de los trabajadores legalmente vinculados, sea directamente con las empresas del sector azucarero o por subcontratación, contrastan significativamente con las de los trabajadores informales.

Los trabajadores informales no solo se encuentran bajo el incumplimiento del marco normativo, también bajo la precarización laboral que incluye pagos dependientes de trabajo y productividad; largas jornadas de trabajo que incluyen el tiempo para desplazarse hasta el trabajo y regresar a sus hogares, lo que afecta la mediación entre trabajo y tiempo con la familia; salarios por debajo del SMMLV que no garantizan condiciones dignas para vivir; experiencias de humillación y atropello de derechos humanos; adultos mayores que asumen tareas que demandan gran esfuerzo físico; trabajadores que no aspiran a una pensión; carencia de dotación y elementos de seguridad, entre otras. Pese a que el vínculo laboral formal y directo ayuda a que el trabajador tenga mayores características de trabajo decente, en algunos casos como en las oportunidades y trato estas condiciones son igual de indecentes que en los trabajadores con vínculo informal.

La palabra indecente utilizada para analizar la ausencia de condiciones decentes, permitió evidenciar la precariedad de este trabajo en sus múltiples dimensiones y la coexistencia de 10 decente y no decente en los trabajos agrícolas de la caña de azúcar, sin importar el tipo de vinculo laboral. Desde una perspectiva de género, se evidencia que esta precarización se acentúa en las mujeres, quienes tienen baja participación en estos trabajos y son excluidas en algunos cultivos de caña de azúcar. Por su rol social de madres y esposas, tienen dobles jornadas de trabajo, deben levantarse antes que los hombres que realizan los mismos trabajos para preparar sus alimentos, ir a trabajar y regresar a sus trabajos domésticos. La mayoría de ellas ingresan a dicha actividad debido a la ausencia de un hombre proveedor de recursos económicos en sus hogares o porque estos recursos no son suficientes.

Pese a que desde el 2008 los trabajadores agrícolas manifestaron y evidenciaron estas condiciones de precarización laboral, el contexto sigue caracterizado por el alto índice de informalidad, lo que implica condiciones de vulnerabilidad para los trabajadores y continuidad en los ciclos de pobreza. Adicional a ello, estos trabajos agrícolas se están reemplazando con máquinas, principalmente en los ingenios, es decir los protagonistas del sector azucarero en la región. Esto implica un declive en la mano de obra directa y formalmente vinculada, y la disponibilidad y aceptación de trabajos informales y precarios en los que todavía no se ha incorporado la máquina para el trabajo agrícola, como en los trapiches. 
Lo anterior lleva a señalar que en el contexto de la fase agrícola de la explotación de la caña de azúcar coexisten trabajos decentes e indecentes, los primeros están mediados por el cumplimiento del marco normativo mientras que los segundos evidencian un proceso de configuración histórica, desde la época de la colonia con el uso de la mano de obra esclava, hasta los efectos de las políticas del neoliberalismo materializados en las CTA y la ausencia de regulación por parte del gobierno, es decir los "contratistas" que vinculan a gran parte de los trabajadores agrícolas de la caña de azúcar.

A partir de una apuesta cualitativa esta investigación puso de manifiesto unas de las características de precarización laboral en las que viven algunos trabajadores y que hacen que sus trabajos sean indecentes. No obstante, se presentaron algunas limitaciones metodológicas, como la disposición del sector empresarial para participar en la investigación y de algunos trabajadores agrícolas que pudieron otorgar más información. Es por ello que, para futuras investigaciones, se recomienda contrastar la información de los trabajadores agrícolas con las cifras de las empresas del sector azucarero, en especial, los ingenios, y obtener así una caracterización cuantitativa de la cantidad de trabajadores que aún continúan con ellas, así como sus datos sociodemográficos.

La investigación, desde una perspectiva cualitativa y con un posicionamiento crítico plantea la necesidad de estrategias de intervención en el sector empresarial - así sean de RSE- para contribuir a las condiciones de trabajo y calidad de vida de los trabajadores agrícolas y fortalecer las apuestas para el logro del objetivo n.8. del trabajo decente y crecimiento económico de la Agenda 2030 (OIT, 2019a).

\section{Referencias}

Anker, R., Chernyshev, I., Egger, P., Mehran, F., y Ritter, J. (2002). Measuring Decent Work with Statistical Indicators. Working Paper No. 2. International
Labour Office. http://lo.ch/wcmsp5/groups/ public/---dgreports/---integration/documents/ publication/wcms_079089.pdf

Antunes, R. (2000). La centralidad del trabajo hoy. Papeles de Población, (25), 83-96.

Asocaña. (2019a). Aspectos generales del sector agroindustrial de la caña 2018-2019. Informe anual. https://wwww.asocana.org/modules/ documentos/15331.aspx

Asocaña. (2019b). El Sector azucarero colombiano en la actualidad. https://wuww.asocana.org/publico/ info.aspx?Cid=215

Bedoya, C. (2017). Configuración del Mundo del Trabajo: Un panorama en Latinoamérica y Colombia. Dimensión Empresarial, 15(1), 77-88.

Bedoya, C. (2019). Historias "subordinadas" en el sector azucarero del Valle del Cauca, Colombia: análisis del Cortero de Caña en las prácticas de RSE. En $54^{\circ}$ Asamblea Anual CLADEA. Lima (Perú).

Dinero. (2007). Sector azucarero es una de las empresas adheridas al pacto global de Naciones Unidas. Revista Dinero. https://wuwu.dinero.com/ actualidad/noticias/articulo/sector-azucareroempresas-adheridas-pacto-global-nacionesunidas/52365

Falzon, M. A. (2009). Introduction: Multi-sited Ethnography: Theory, Praxis and Locality in Contemporary Research. En Multi-sited ethnography. Theory, Praxis and Locality in Contemporary Research (pp. 1-23). Ashgate Publishing Limited.

FAO, Organización de las Naciones Unidas para la Alimentación y la Agricultura. (2013). Guidance on how to address decent rural employment in FAO country activities FAO country activities 
(2ed.). Food and Agriculture Organization of the United Nations (FAO).

Flick, U. (2007). Designing Qualitative Research. Sage Publications.

Giraldo, P. C. (2017). La flexibilización multidimensional de los trabajadores cosecheros de la caña de azúcar: situación laboral y social de un grupo de corteros del Valle del Cauca, Colombia. Ulúa, 29, 191-225.

Guba, E., y Lincoln, Y. S. (1994). Competing Paradigms in Qualitative Research. En N. K. Denzin y Y. S. Lincoln (eds.), Handbook of Qualitative Research (pp. 105-117). Sage Publications.

Jaramillo, J. E. (2017). Movilización de los corteros de caña de azúcar en el Valle del Cauca, huellas y despliegues de una acción colectiva. Revista de Antropología y Sociología, 19(2), 93-114.

Marcus, G. E. (2001). Etnografía en/del sistema mundo. El surgimiento de la etnografía multilocal. Alteridades, 11(22), 111-127.

Mayan, M. J. (2009). Essentials of Qualitative Inquiry. Left Coast Press.

Montoya, G. (2011). El paro de corteros de caña en el Valle del Cauca - Colombia: Una acción colectiva de cara al modelo económico. Entramado, 7(1), 104-114. http://wuruw.scielo.org.co/pdf/entra/v7n1/ v7ח1ว07.pdf

Organización Internacional del Trabajo (OIT). (2013). Decent work indicators. Guidelines for producers and users of statistical and legal framework indicators (2 ed.). International Labour Organization.

Organización Internacional del Trabajo (OIT). (2018). Sustainable Development Goals: A Guidebook on SDG Labour Market Indicators. International Labour Organization.

Organización Internacional del Trabajo (OIT) (2019a). Objetivo \#8: Trabajo decente y crecimiento económico. https://wuw ilo.org/global/topics/ sog-2030/goal-8/lang--es/index.htm

Organización Internacional del Trabajo (OIT) (2019b). Trabajo decente y productivo en la agricultura. OIT.

Organización Internacional del Trabajo (OIT). (2020). The ILO's Decent Work Agenda. https://wwwwilo.org/ global/topics/decent-work/lang--en/index.htm

Organización Internacional del Trabajo (OIT) y Monje, L. M. (2014). Trabajo decente, pobreza y empleo agricola. Experiencias de organización sindical en el sector rural frente a la informalidad en América Latina. Centro Internacional de Formación de la OlT.

Oya, C. (2015). Decent Work Indicators for agriculture and rural areas: Conceptual issues, data collection challenges and possible areas for improvement (No. 15-10). ESS Working Paper. FAO. www.fao. org/3/a-i5060e.pdf

Patton, M. (2015). Qualitative Research \& Evaluation Methods (4 ed.). Thousand Sage Publication.

Pérez-Rincón, M. A., y Alvarez-Roa, P. (2009). Deuda social y ambiental del negocio de la caña de azúcar: Responsabilidad social empresarial y subsidios implícitos en la industria cañera. ARFo Editores e Impresores.

Ronderos, C., y Palacios, L. (2010). Aspectos económicos, sociales y ambientales de la industria de la caña de azúcar en Colombia. Universidad Sergio Arboleda.

Sánchez, R. (2008). Las iras del azúcar: la huelga de 1976 en el Ingenio Riopaila. História Crítica, (35), 34-57. 
Saukko, P. (2012). Metodologías para los estudios culturales. En N. K. Denzin y Y. S. Lincoln (eds.), Paradigmas y perspectivas en disputa (pp. 316-340). Editorial Gedisa.

Soto, Á. (2008). Flexibilidad laboral y subjetividades. Hacia una comprensión psicosocial del empleo contemporáneo. Editorial LOM.

Soto, Á., Espinoza, G., y Gómez, J. (2008). Aspectos subjetivos vinculados a la flexibilidad laboral. in Flexibilidad laboral y subjetividades. Hacia una comprensión psicosocial del empleo contemporáneo (pp. 11-37). LOM Ediciones y Universidad Alberto Hurtado.
Strauss, A., y Corbin, J. (2002). Bases de la investigación cualitativa. Técnicas y procedimientos para desarrollar la teoría fundamentada. Editorial Universidad de Antioquia.

Sustainable Develpment Goals Fund (SDGF). (2019). Alcanzar los Objetivos de Desarrollo Sostenible. https://wwwu.sogfund.org/es

Willig, C. (2001). Introducing Qualitative Research in Psychology. Adventures in theory and method. Open University Press, McGraw-Hill Education y McGraw-Hill House. 